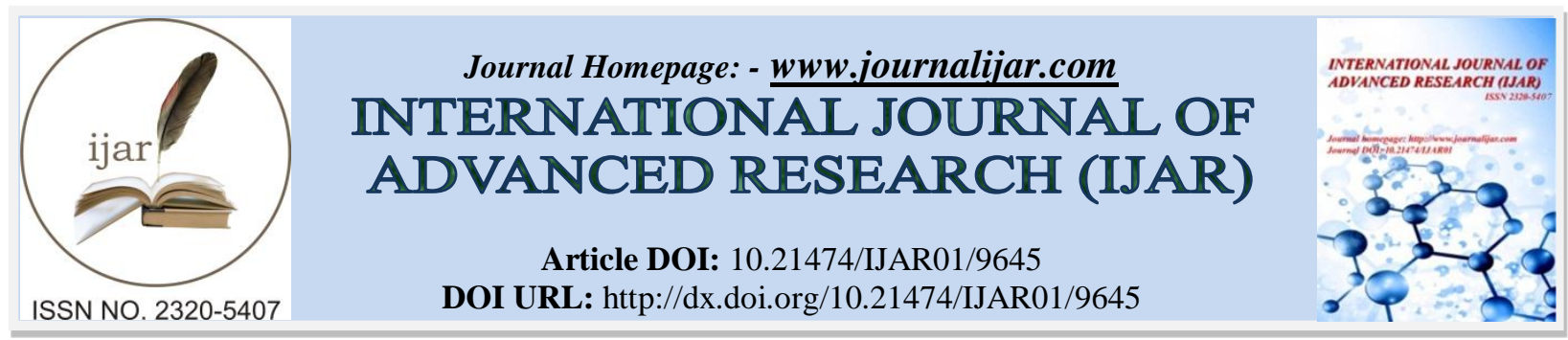

RESEARCH ARTICLE

\title{
ROLE OF DIFFUSION WEIGHTED MRI IN EVALUATION OF BIRADS3 AND BIRADS4 BREAST LESIONS.
}

Heba Ahmed Mostafa ${ }^{1}$, Sahar Mansour ${ }^{2}$, Sahar M abd Elsalam ${ }^{1}$ and Soha.t.Hamed ${ }^{2}$.

1. Department of Radiology, Faculty of Medicine, Beni suef University, Egypt.

2. Department of Radiology, Faculty of Medicine, Cairo University, Egypt.

\section{Manuscript Info}

Manuscript History

Received: 04 July 2019

Final Accepted: 06 August 2019

Published: September 2019

Key words:-

Breast cancer, DCE-MRI, DWI, BIRADS, Sensitivity.

\begin{abstract}
Background: Precise diagnosis of suspicious breast lesions helps in better assessment and management. The aim of this study is to explore the role of DCE-MRI and DWI in evaluation of suspicious breast lesions (BI-RADS 3 and 4).

Subjects and Methods: A total of 50 patients with suspicious breast lesions (BI-RADS 3 and 4) participated in the study. After giving informed consent, all patients were subjected to DWI and DCE-MRI, and their findings were compared to histopathology results. DCE-MRI curves, borders, and enhancement were described, and DWI findings were also extracted. Factors predicting distinction of cancerous tissue from benign tissue by DCE-MRI and DWI according to histopathology reference have been analyzed.

Results: Using DCE-MRI, out of 19 patients proven by histopathology to have benign lesions, 12 (63.2\%) showed mass lesions and out of 29 patients with malignant lesions $21(72.4 \%)$ had mass lesions $(\mathrm{p}<0.001)$.Curves of benign lesions were mostly persistent $(83.3 \%)$ whenever malignant curves were either plateau or wash out;(47.6\%) each $(\mathrm{p}<0.001)$. ADC brightness was more frequent in benign lesions $(p<0.05)$. The best cut-off of ADC that can differentiate between malignant and benign lesions was 1.19 (sensitivity 94.7\% and specificity $67.7 \%$ ).DCE-MRI had a sensitivity of $75.8 \%$ and specificity of $73.7 \%$, while DWI had a sensitivity of $82.8 \%$ and specificity of $73.7 \%$. No statistically significant differences have been noticed between benign and malignant breast non-mass lesions regarding any of the studied measures ( $p>0.05$ ).

Conclusion: DCE-MRI and DWI are crucial in distinction of suspicious breast mass lesions. The sensitivity and specificity of DCEMRI and DWI as diagnostics have been determined.
\end{abstract}

Copy Right, IJAR, 2019, All rights reserved.

\section{Introduction:-}

Magnetic resonance imaging (MRI) of breast is suggested to be adjunct to mammography in screening women with higher risk to develop breast cancer. ${ }^{1}$ Although mammography is crucial in clinical practice especially in assessment of nodal status and mass sizes, and guiding needle biopsies, its function is restricted by lower sensitivity, presence of dense tissue and architectural distortion that could mask the detection of breast cancer. ${ }^{2}$ On the other hand, 
dynamic contrast-enhanced MRI (DCE-MRI)and diffusion-weighted imaging (DWI) have the ability to differentiate between normal and diseased breast tissue with higher sensitivity. Such distinction could minimize the number of biopsies, especially in suspicious lesions. Therefore it is gaining popularity in clinical practice as a complementary diagnostic tool for breast cancer. ${ }^{3}$

Breast DCE-MRI includes typically multiple sequences of post-contrast recorded at different times after injecting a contrast agent. The time-signal intensity curves resulting from these sequences reflect the intensity changes induced by contrast uptake over different periods. These kinetic curves can be categorized according to the voxel level to persistent, plateau and washout, and may helpin diagnosing breast cancer, expecting cancer recurrence, and measuring chemotherapeutic response. Other kinetic characteristics such as enhancement pattern in addition to shape of lesion may be of diagnostic value in detecting breast cancer and its subtypes. ${ }^{4-8}$

However, specificity of DCE-MRI is still questionablein screeningand characterization, because the interpretation of breast DCE-MRI dependson many factors such as expertise ofthe radiologistand adequate use of visualization techniques, which could result in substantialamount of false positive results. ${ }^{8,9}$ Further, DCE-MRI is an expensive technique, and could not be available in most health-settings. Also, it cannot be used for patients with previous reaction to contrast agents or those with renal dysfunction. 8,9

On the other hand, DWI measures the mobility of water in tissue. The movement of water is more restricted in tissues with a higher cellular content and numerous intact cell membranes such as those in malignant breast lesions. DWI is considered sensitive to disrupted characteristics that could indicate malignancysuch as cell organization, density, extracellular space, and cell membrane permeability. This advantage may help inmore precise discrimination between benign and cancerous breast lesions. ${ }^{8}$

In this regards, the objective of this study is to investigate the role of DCE-MRI and DWI in evaluation of suspicious breast lesions (BI-RADS 3 and 4).

\section{Subjects and Methods:-}

In this observational study, a total of 50 patients withsuspicious breast lesions, andcategorized according to Breast Imaging Reporting and Data System (BI-RADS) as BI-RADS3 and 4, usingfull-field digital mammography and ultrasonography, were recruited for this study. Exclusioncriteria were history of breast implants, lactating, benign breast surgery within 1 year, and ineligibility for breast MRI.

Then, all included patients were referred for further DWI and DCE-MRI examination.For premenopausal women; MRI was ideallyscheduled the $2^{\text {nd }}$ week of the menstrual cycle.

DWI was performed before the DCE-MRI acquisition using a diffusion-weighted echo-planar imaging (EPI) sequence with parallel imaging (Philips Intera); reduction factor 2, 7000/71.5,number of excitations 2 , matrix $240 \times$ 240, field of view $34 \mathrm{~cm}$, slice thickness $3 \mathrm{~mm}$, and gap 0. Diffusion gradients were applied in 6 directions with b = $0,300,600$ and $1500 \mathrm{~s} / \mathrm{mm}^{2}$, and the scanning time was 4 minutes. Respiratory triggering was used for better resolution. ${ }^{9,10}$

A bolus of gadolinium-dimeglumine (Gd-DTPA) (Magnavist,Schering AG Berlin,Germany) was injected manually intravenous at a dose of $(0.1 \mathrm{mmol} / \mathrm{kg})$ followed by saline flush to ensure that contrast-enhanced images could be obtained immediately after contrast agent injection. ${ }^{9,10}$

Dynamic T1 WIs wasperformed, using Gradient echo T1-weighted image, with fat suppression at the following time points: 1.27 minute, 2.55 minute, 4.21 minute, 5.47 minute and 7.13 minute. ${ }^{9,10}$

Post processing image subtraction was obtained between the post contrast imaging showing maximum enhancement and pre-contrast images (in the same axial plane).Diffusion maps were formed. A noise-level threshold of 200 was applied to mask the $\mathrm{b}=0 \mathrm{~s} / \mathrm{mm}^{2}$ images before forming diffusion maps, andapparent diffusion coefficient (ADC) maps were obtained from the resulting DWIs. ${ }^{9,10}$

Then, DCE-MRI was performed with high field strength 1.5 Tesla on Signa system (Philips Intera) using dedicated double breast coil.Coronal T1 weighted spin echo sequence was carried out for localization purpose and followed by 
plain sequences using $\mathrm{T} 1$-weighted fast spin echo sequence ( $\mathrm{TR}=501 \mathrm{msec}$., $\mathrm{TE}=10 \mathrm{msec}$.), in addition to $\mathrm{T} 2$ weighted fast spin echo sequence (TR=4131mesc., $T E=120 \mathrm{msec}$.) in axial orientation. ${ }^{5}$

For each DCE-MRI-detected lesion at the corresponding location on the combined DWI series, aregion of interest (ROI) was defined. The mean ADC of the voxels in the ROI was calculated for each lesion. Quantitative analysis was done by placing the ROI at the most enhanced part within the lesion result in automatically created time/signal intensity curve. ${ }^{10}$

Subtraction images were first examined to detect the presence or absence of lesion enhancement.In case of lesion enhancement the corresponding non subtracted pre-contrast and post contrast images in each time point was viewed together and lesions interpretation took place whether it a focus, mass or non-mass like enhancement.In case of mass enhancement evaluation was carried out as follow; shape, border, pattern of enhancement and dynamic behavior. In case of non-mass like enhancement, its distribution and enhancement pattern were evaluated. ${ }^{5,10}$

DWIs and ADC maps were then examined regarding the signal intensity and the mean ADC of each lesion. BIRADS classification was applied for each lesion based on the combination of morphologic and kinetic criteria. ${ }^{5,10}$ Later,a surgical biopsy was performed for all patients and those with non-palpablelesions underwent radio-guided occult lesion localization (ROLL) prior to the biopsy. Haematoxylin and eosinstained slides of formalin-fixed and paraffin-embeddedtissue blocks were assessed by a pathologist.

The study was conducted in Kasr El-AiniHospital during the period between December 2015 and January 2017. Data entry, verification, and validation were carried out using standard computer software. Data were analyzed using the software, Statistical Package for Social Science (SPSS Inc. Released 2009, PASW Statistics for Windows, version 18.0: SPSS Inc., Chicago, Illinois, USA), then processed and tabulated. Frequency distribution with its percentage and descriptive statistics with mean and standard deviation were calculated. Chi-square, t-test, correlations were done whenever needed. $\mathrm{P}$ values of less than 0.05 were considered significant.

The study was conducted in full accordance with the guidelines for Good Clinical Practice and the Declaration of Helsinki, and data for each patient were collected onlyafter obtaining that patient's signed written data release forms.

\section{Results:-}

Out of the 50 included patients with suspicious breast lesions, 2 patients were considered free by both MRI and histopathology.

DCE-MRI showed that out of the 19 patients proven by histopathology to have benign lesions, 12 (63.2\%) showed mass lesions and out of 29 patients with malignant lesions 21 (72.4\%) had mass lesions, while focal lesions were more associated with benign lesions $(\mathrm{p}<0.001)$. Malignant lesions were more likely to show Type III curves; $41.4 \%$ compared to $0 \%$ in benign lesions ( $\mathrm{p}<0.001)$ (Table 1).

For mass lesions, the margins of the benign lesions tended to be distinct (91.7\%), while those of the malignant lesions were either indistinct $(66.7 \%)$ or speculated $(23.8 \%)$ ( $\mathrm{p}<0.001)$. Benign lesions were more likely to show homogenous enhancement (66.8\%), while malignant lesions were more heterogeneous $(47.7 \%)$ ( $\mathrm{p}<0.05)$. Curves of benign lesions were mostly persistent $(83.3 \%)$ whenever malignant curves were either plateau or wash out; $(47.6 \%)$ each $(\mathrm{p}<0.001)$. Further, ADC was significantly higher in benign lesions compared to malignant lesions, and the brightness was more frequent in benign lesions $(\mathrm{p}<0.05)$. Also, DWI of mass lesions showed that benign lesions were more likely to be bright $(\mathrm{p}<0.05)($ Table 1$)$.

On the other hand, no statistically significant differences have been noticed between histopathology-proven benign and malignant lesions regarding enhancement, curve shape ADC or DWI ( $p>0.05)$ (Table 1).

However, DCE-MRI and DWI did not show good ability in distinction between non-mass cancerous and benign lesions, since no statistically significant differences have been shown between both groups ( $>0.05$ ) (Table 1).

DCE-MRI was accurate in diagnosing $75 \%$ of lesions with a sensitivity of $75.8 \%$ and specificity of $73.7 \%$, while DWI achieved an accuracy of $77.6 \%$ with a sensitivity of $82.8 \%$ and specificity of $73.7 \%$ (Table 2 ). 
The best cut-off of ADC that can differentiate between malignant and benign lesions stood at 1.19 (AUC 0.83, sensitivity $94.7 \%$, and specificity $67.7 \%$ ) (Figure 1).

Table 1:-Assessment of MRI findings in regards to histopathological findings

\begin{tabular}{|c|c|c|c|c|}
\hline \multirow{2}{*}{\multicolumn{2}{|c|}{ MRI }} & \multicolumn{3}{|c|}{ Histopathology } \\
\hline & & Benign (n, \%) & Malignant (n, \%) & P value \\
\hline \multirow[t]{3}{*}{ Morphology $(\mathrm{n}=48)$} & Mass & $12(63.2)$ & $21(72.4)$ & \multirow{3}{*}{$<0.001$} \\
\hline & Non-mass & $4(21.0)$ & $7(24.1)$ & \\
\hline & Focal areas & $3(15.8)$ & $1(3.5)$ & \\
\hline \multirow[t]{3}{*}{ Curve $(\mathrm{n}=48)$} & Type I & $11(57.9)$ & $5(17.2)$ & \multirow[t]{3}{*}{$<0.001^{*}$} \\
\hline & Type II & $8(42.1)$ & $12(41.4)$ & \\
\hline & Type III & $0(0.0)$ & $12(41.4)$ & \\
\hline \multirow{3}{*}{$\begin{array}{l}\text { Mass Margin } \\
(\mathrm{n}=33)\end{array}$} & Distinct & $11(91.7)$ & $2(9.5)$ & \multirow[t]{3}{*}{$<0.001^{*}$} \\
\hline & Indistinct & $1(8.3)$ & $14(66.7)$ & \\
\hline & Speculated & $0(0.0)$ & $5(23.8)$ & \\
\hline \multirow{4}{*}{$\begin{array}{l}\text { Mass Enhancement } \\
\text { Pattern }(n=33)\end{array}$} & No & $2(16.6)$ & $0(0.0)$ & \multirow{4}{*}{$0.024 *$} \\
\hline & Homogenous & $8(66.8)$ & $7(33.3)$ & \\
\hline & Heterogeneous & $1(8.3)$ & $10(47.7)$ & \\
\hline & Ring & $1(8.3)$ & $4(19.0)$ & \\
\hline \multirow{3}{*}{$\begin{array}{l}\text { Mass Curve } \\
\text { Shape }(n=33)\end{array}$} & Persistent & $10(83.3)$ & $1(4.8)$ & \multirow{3}{*}{$<0.001 *$} \\
\hline & Plateau & $2(16.7)$ & $10(47.6)$ & \\
\hline & Wash out & $0(0.0)$ & $10(47.6)$ & \\
\hline \multirow[t]{2}{*}{ Mass Diffusion $(\mathrm{n}=33)$} & Restricted & $8(66.8)$ & $16(76.2)$ & \multirow{2}{*}{0.762} \\
\hline & Facilitated & $4(33.2)$ & $5(23.8)$ & \\
\hline \multicolumn{2}{|c|}{ Mass Quantitative ADC $($ Mean \pm Sd) } & $1.3 \pm 0.4$ & $1.0 \pm 0.3$ & $0.048^{*}$ \\
\hline \multirow{4}{*}{$\begin{array}{l}\text { Mass Qualitative } \\
\text { ADC }(n=33)\end{array}$} & Bright & $7(58.3)$ & $1(4.8)$ & \multirow[t]{4}{*}{$0.001^{*}$} \\
\hline & Intermediate & $3(25.0)$ & $13(61.9)$ & \\
\hline & $\begin{array}{l}\text { Intermediate- } \\
\text { Bright }\end{array}$ & $2(16.7)$ & $0(0.0)$ & \\
\hline & Low & $0(0.0)$ & $7(33.3)$ & \\
\hline \multirow[t]{3}{*}{ Mass DWI $(\mathrm{n}=33)$} & Bright & $8(66.6)$ & $0(0.0)$ & \multirow[t]{3}{*}{0.123} \\
\hline & Intermediate & $2(16.7)$ & $16(76.2)$ & \\
\hline & $\begin{array}{l}\text { Intermediate- } \\
\text { Bright }\end{array}$ & $2(16.7)$ & $5(23.8)$ & \\
\hline \multirow{2}{*}{$\begin{array}{l}\text { Non-mass Enhancement } \\
\text { Pattern }(\mathrm{n}=11)\end{array}$} & Homogenous & $2(50.0)$ & $1(14.3)$ & \multirow[t]{2}{*}{0.279} \\
\hline & Heterogeneous & $2(50.0)$ & $6(85.7)$ & \\
\hline \multirow{3}{*}{$\begin{array}{l}\text { Non-mass Curve } \\
\text { Shape }(n=33)\end{array}$} & Persistent & $0(0.0)$ & $3(42.8)$ & \multirow[t]{3}{*}{0.073} \\
\hline & Plateau & $4(100.0)$ & $2(28.6)$ & \\
\hline & Wash out & $0(0.0)$ & $2(28.6)$ & \\
\hline \multicolumn{2}{|c|}{ Non-mass Quantitative ADC (Mean \pm Sd) } & $1.4 \pm 0.1$ & $1.3 \pm 0.3$ & \multirow{4}{*}{$\begin{array}{l}0.521 \\
0.378\end{array}$} \\
\hline \multirow{3}{*}{$\begin{array}{l}\text { Non-mass Qualitative } \\
\operatorname{ADC}(n=11)\end{array}$} & Bright & $2(50.0)$ & $1(14.3)$ & \\
\hline & Intermediate & $2(50.0)$ & $5(71.4)$ & \\
\hline & Low & $0(0.0)$ & $1(14.3)$ & \\
\hline \multirow[t]{2}{*}{ Non-mass DWI (n=11) } & Bright & $3(75.0)$ & $5(71.4)$ & 0.721 \\
\hline & Intermediate & $1(25.0)$ & $2(28.6)$ & \\
\hline
\end{tabular}

*p value is considered significant

Table 2:-The diagnostic accuracy of MRI compared to histopathological findings

\begin{tabular}{|c|c|c|c|c|}
\hline \multicolumn{2}{|c|}{ Accuracy } & \multicolumn{3}{|c|}{ Histopathology } \\
\hline & & Benign & Malignant & Total \\
\hline \multirow[t]{3}{*}{ DCE-MRI } & Benign & $14(\mathrm{TN})$ & $7(\mathrm{FN})$ & 21 \\
\hline & Malignant & $5(\mathrm{FP})$ & 22 (TP) & 27 \\
\hline & Total & 19 & 29 & 48 \\
\hline \multicolumn{4}{|c|}{ Sensitivity=TP/TP+FN= } & $75.8 \%$ \\
\hline
\end{tabular}




\begin{tabular}{|c|c|c|c|c|}
\hline \multicolumn{4}{|c|}{ Specificity=TN/TN+FP= } & $73.7 \%$ \\
\hline \multicolumn{4}{|c|}{ Positive Predictive Value $=\mathrm{TP} / \mathrm{TP}+\mathrm{FP}=$} & $81.5 \%$ \\
\hline \multicolumn{4}{|c|}{ Negative Predictive Value $=\mathrm{TN} / \mathrm{TN}+\mathrm{FN}=$} & $66.7 \%$ \\
\hline \multicolumn{4}{|c|}{ Accuracy=TP+TN/TP+TN+FP+FN= } & $75 \%$ \\
\hline \multirow{2}{*}{\multicolumn{2}{|c|}{ Accuracy }} & \multicolumn{3}{|c|}{ Histopathology } \\
\hline & & Benign & Malignant & Total \\
\hline \multirow[t]{3}{*}{ DWI-MRI } & Benign & $14(\mathrm{TN})$ & $5(\mathrm{FN})$ & 19 \\
\hline & Malignant & $5(\mathrm{FP})$ & $24(\mathrm{TP})$ & 29 \\
\hline & Total & 19 & 29 & 48 \\
\hline \multicolumn{4}{|c|}{ Sensitivity $=\mathrm{TP} / \mathrm{TP}+\mathrm{FN}=$} & $82.8 \%$ \\
\hline \multicolumn{4}{|c|}{ Specificity $=\mathrm{TN} / \mathrm{TN}+\mathrm{FP}=$} & $73.7 \%$ \\
\hline \multicolumn{4}{|c|}{ Positive Predictive Value $=\mathrm{TP} / \mathrm{TP}+\mathrm{FP}=$} & $82.8 \%$ \\
\hline \multicolumn{4}{|c|}{ Negative Predictive Value $=\mathrm{TN} / \mathrm{TN}+\mathrm{FN}=$} & $73.7 \%$ \\
\hline \multicolumn{4}{|c|}{ Accuracy $=\mathrm{TP}+\mathrm{TN} / \mathrm{TP}+\mathrm{TN}+\mathrm{FP}+\mathrm{FN}=$} & $77.6 \%$ \\
\hline
\end{tabular}

TP; True Positive, TN; True Negative, FP; False Positive, FN; False Negative

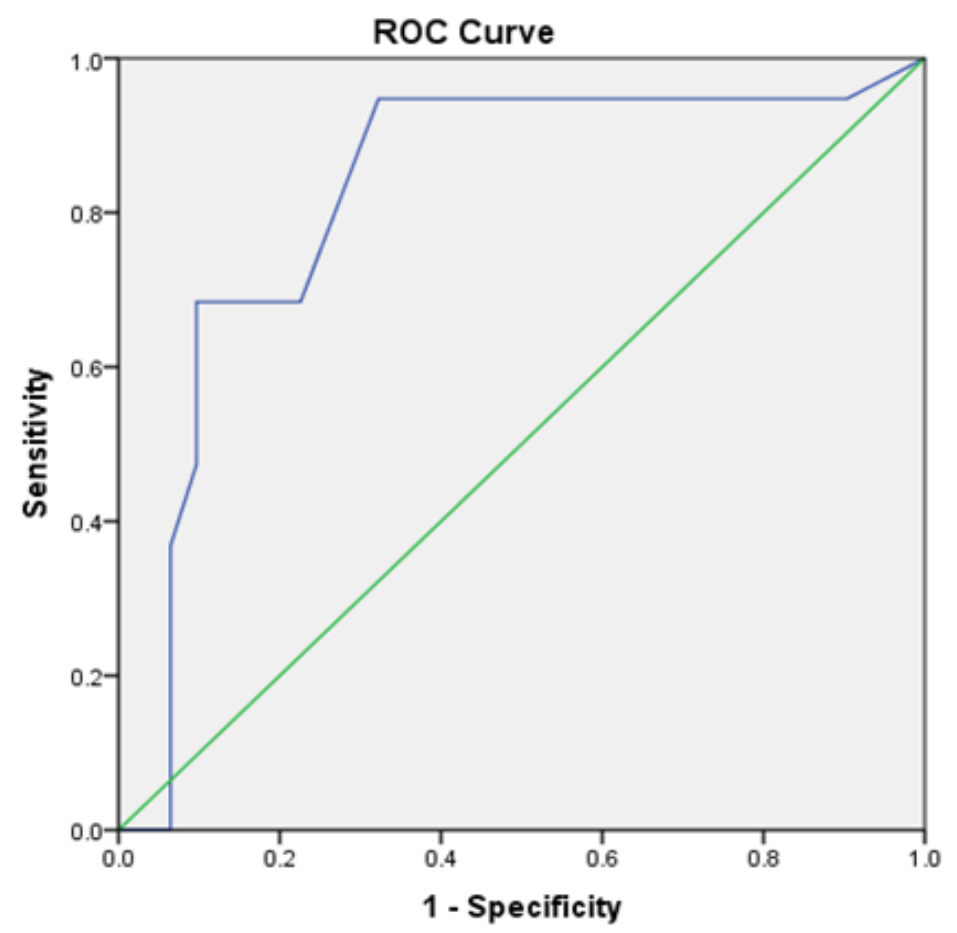

Area under the curve (AUC): 0.83; 95\%CI 0.70-0.95; Best Cut-off:1.19; Sensitivity: 94.7\%, Specificity: 67.7\%

Figure 1:-ROC curve for the best cut-off diagnostic value of ADC to malignancy

\section{Discussion:-}

Identifying precise and effective tools in screening for breast cancer in suspicious lesions is crucial. In the current study, the characteristics of cancerous mass lesions have been clearly differentiated from benign lesions, using DCEMRI and DWI; due to the differences in type of kinetic curves, enhancement patterns, margins and borders.Both quantitative $\mathrm{ADC}$ and qualitative $\mathrm{ADC}$ have been also shown as predictors for cancerous lesions. Malignant lesions in our study were more likely to take mass shape and type III kinetic curves. Malignant mass lesions were commonly of indistinct margin, heterogeneous enhancement and plateau or washout curves. Quantitative ADC of malignant mass lesions was lower and qualitative ADC was less bright, compared to benign lesions.Also, when compared to histopathology as a reference DCE-MRI had a sensitivity of $75.8 \%$ and specificity of $73.7 \%$, while DWI had a sensitivity of $82.8 \%$ and specificity of $73.7 \%$. 
In consistence with our findings, a recent systematic review over several studies using DCE-MRI for diagnosis of breast cancer showed that the kinetic and morphological features of mass lesions described by DCE-MRI can differentiate betweencancerous and benign lesions with reasonable sensitivity and specificity. ${ }^{11}$

A recent retrospective study over 51 patients with breast cancer and 51 controls showed that kinetic measures of DEC-MRI could distinct between breast cancer and benign lesions. The authors also concluded that the abnormal enhancement characteristics may represent biological progression of potentialbreast cancer. ${ }^{12}$

Other studies have also focused on the role of the morphological features, detected by DCE-MRI, in diagnosing breast cancer. The studies showed in details the indication of DCE-MRI in identifying and evaluating breast lesions, especially in high risk patients. ${ }^{13,14}$

Also, DWI has been suggested to be useful in assessing the malignant suspicious mass lesions detected on screening mammograms and reduce unnecessary biopsies.DWI improves the diagnostic accuracy of breast MRI, since it provides information about the cellularity of thelesion. ${ }^{9}$

The accuracy of MRI in diagnosing suspicious lesions have been investigated bySardanelli and colleagues whoexamined results of 19 patients with suspicious mammographic findings; 5 of these were malignant, 4 of which could be diagnosed by MRI achieving a sensitivity of $80 \% .^{15}$

Alike, Lee et al cited outcomes of MRI for 86 suspicious lesions, 9of them were malignant; and there was 1 additional incidental malignancy reported among a total 12 MRI lesions. ${ }^{16}$ Similar studies on suspicious lesions, however on different sample sizes, have also reached the same conclusion. ${ }^{17-19}$

In the current study, we could not find statistically significant differences between benign and malignant non-mass breast lesions regarding morphological or enhancement kinetic features of DCE-MRI or DWI.

In agreement, a recently-published study over patients with non-mass lesions showed that DCE-MRI could not differentiate for sure between malignant and benign non-mass lesions. The authors concluded that DCE-MRI cannot show the subtle histological differencesbetween non-proliferative lesions, proliferativelesions without atypia and proliferative lesionswith atypia. They also recommended further histopathology specimens for such cases. ${ }^{20}$ In contrary, DWI in one study was more specific than DCE-MRI in defining the nature of non-mass lesions. ${ }^{21}$

In our study, ADC values of benign mass lesions were significantly higher than those of malignant mass lesions. Previous studies have suggested the efficacy of ADC in the precise differentiation between benign and malignant breast lesions, yielding different calculated ADC values and cutoff points. ${ }^{6,22}$

In conclusion, DCE-MRI and DWI are essential in distinction of suspicious breast mass lesions; however their ability to differentiate between malignant and benign non-mass lesions is still questionable. The sensitivity and specificity of DCE-MRI and DWI are comparable and their diagnostic abilityhas been determined.

\section{References:-}

1. Saslow D, Boetes C, Burke W, Harms S, Leach MO, Lehman CD, et al.American Cancer Society guidelines for breast screening with MRI as anadjunct to mammography. Cancer J Clin. 2007; 57:75-89.

2. Boyd NF, Martin LJ, Yaffe MJ, Minkin S. Mammographic density and breastcancer risk: current understanding and future prospects. Breast Cancer Res.2011; 13(6):223.

3. Weinstein S, Rosen M. Breast MR imaging: current indications and advancedimaging techniques. RadiolClin $\mathrm{N}$ Am. 2010; 48(5):1013-42.

4. Hylton N. Dynamic contrast-enhanced magnetic resonance imaging as animaging biomarker. J ClinOncol. 2006; 24(20):3293-8.

5. Kuhl CK, Mielcareck P, Klaschik S, Leutner C, Wardelmann E, Gieseke J, et al.Dynamic breast MR imaging: are signal intensity time course data useful fordifferential diagnosis of enhancing lesions? Radiology.1999; 211(1):101-10.

6. El Khouli RH, Macura KJ, Jacobs MA, Khalil TH, Kamel IR, Dwyer A, et al.Dynamic contrast-enhanced MRI of the breast: quantitative method forkinetic curve type assessment. Am J Roentgenol. 2009; 193(4):295-300. 
7. Partridge SC, Rahbar H, Murthy R, Chai X, Kurland BF, DeMartini WB, et al.Improved diagnostic accuracy of breast MRI through combined apparentdiffusion coefficients and dynamic contrast-enhanced kinetics. MagnResonMed. 2011; 65(6):1759-67.

8. Krammer J, Pinker-Domenig K, Robson ME, Gonen M, Bernard-Davila B, Morris EA, et al. Breast cancer detection and tumor characteristics in BRCA1 and BRCA2 mutation carriers. Breast Cancer Res Treat. 2017; 163:565-71.

9. Mann RM., Kuhl CK, Kinkel K, Boetes C. Breast MRI: guidelines from the European society of breast imaging. EurRadiol 2008; 18, 1307-18.

10. Ashraf AB, Gavenonis SC, Daye D, Mies C, Rosen MA, Kontos D. A multichannelMarkov random field framework for tumor segmentation with an applicationto classification of gene expression-based breast cancer recurrence risk. IEEETrans Med Imaging. 2013; 32(4):637-48.

11. Fusco R, Sansone M, Filice S, Carone G, Amato DM, Sansone C, et al.Pattern Recognition Approaches for Breast Cancer DCE-MRI Classification: A Systematic Review.J. Med. Biol. Eng. 2016; 36:449-59.

12. Wu S, Berg WA, Zuley ML, Kurland BF, Jankowitz RC, Nishikawa R, et al.Breast MRI contrast enhancement kinetics of normal parenchyma correlate with presence of breast cancer.Breast Cancer Research 2016; 18:76.

13. Lee CH. Problem solving MR imaging of the breast. RadiolClin North Am 2004; 42:919-934.

14. Nunes LW, Schnall MD, Orel SG. Update of breast MR imaging architectural interpretation model. Radiology 2001; 219:484-494.

15. Sardanelli F, Melani E, Ottonello C. Magnetic resonance imaging of the breast in characterizing positive or uncertain mammographic findings. Cancer Detect Prev 1998; 22(1):39-42.

16. Lee CH, Smith RC, Levine JA, Troiano RN, Tocino I. Clinical usefulness of MR imaging of the breast in the evaluation of the problematic mammogram. AJR Am J Roentgenol 1999; 173(5):1323-9.

17. Spick C, Szolar DHM, Preidler KW, Tillich M, Reittner P, Baltzer PA. Breast MRI used as a problem-solving tool reliably excludes malignancy. Eur J Radiol 2015; 84(1):61-4.

18. Oztekin PS, Kosar PN. Magnetic resonance imaging of the breast as a problem-solving method: to be or not to be? Breast J 2014;20 (6):622-31.

19. Strobel K, Schrading S, Hansen NL, Barabasch A, Kuhl CK. Assessment of BI-RADS category 4 lesions detected with screening mammography and screening US: utility of MR imaging. Radiology 2015;274 (2):34351.

20. Milosevic ZC, Nadrljanski MM,Milovanovic ZM, Gusic NZ,Vucicevic SS, Radulovic OS.Breast dynamic contrast enhanced MRI:fibrocystic changes presenting as a non-mass enhancement mimicking malignancyRadiolOncol 2017; 51(2): 130-6.

21. Ochi M, Kuroiwa T, Sunami S, Murakami J, Murakami J, Miyahara S, Nagaie T, et al. Diffusion-weighted imaging ( $\mathrm{b}$ value $=1500 \mathrm{~s} / \mathrm{mm}(2)$ ) is useful to decrease false-positive breast cancer cases due to fibrocystic changes. Breast Cancer 2013; 20: 137-44.

22. Sinha S, Lucas-Quesada FA, Sinha U, DeBruhl N, Bassett LW. In vivo diffusion-weighted MRI of the breast: potential for lesion characterization. J MagnReson Imaging 2002; 15(6):693-704. 